\title{
An Approach to Adaptive Quadratic Structuring Functions Based on the Local Structure Tensor
}

\author{
Anders Landström \\ Luleå University of Technology, Luleå, Sweden \\ anders.p.landstrom@ltu.se
}

\begin{abstract}
Classical morphological image processing, where the same structuring element is used to process the whole image, has its limitations. Consequently, adaptive mathematical morphology is attracting more and more attention. So far, however, the use of non-flat adaptive structuring functions is very limited. This work presents a method for defining quadratic structuring functions from the well known local structure tensor, building on previous work for flat adaptive morphology. The result is a novel approach to adaptive mathematical morphology, suitable for enhancement and linking of directional features in images. Moreover, the presented strategy can be quite efficiently implemented and is easy to use as it relies on just two user-set parameters which are directly related to image measures.
\end{abstract}

Keywords: adaptive morphology, quadratic structuring functions, local structure tensor

\section{Introduction}

\section{$1.1 \quad$ Background}

The aim of this work is to present a method for adaptive non-flat morphological filtering, suitable for enhancing or linking directional features and patterns. Classical morphological operators are non-adaptive, i.e. the whole image is probed by the same structuring element without taking variations in structure into account. This is often not ideal, however. In particular; the resulting morphological operations risk stretching over edges, which may destroy important structure information. This has led to the development of adaptive mathematical morphology, where the structuring element (in the adaptive case often called structuring function) may change for each point in the image. For a review of the field of adaptive mathematical morphology, the interested reader is referred to [9] and [14].

Of particular interest to this work are adaptive methods that rely on image structure, i.e. methods where structuring elements are defined based on edges and contours rather than restricted by e.g. measures of similarity. These methods are suitable for prolonging shapes or bridging gaps, thereby emphasizing directional structures in the processed image. Some methods of this type work 
in multiple scales in order to adapt the size of the structuring element to the local scale of structures in the image $[2,18]$. Other methods address structure by considering local orientation only [17], or by combining local orientation with other factors such as distances to edges [21] or degree of anisotropy [12]. It should be noted, however, that one can always find an orientation even though it may very well be completely irrelevant (i.e. where there is no prevalent orientation). Imposing an orientation in such cases may introduce bias from the method itself. Consider, for instance, the simple case of unintentionally assigning a line of random orientation as structuring element. Hence the degree of anisotropy is an important aspect.

The Local Structure Tensor (LST) is a well known method for representing image structure, containing information about both local orientation and degree of anisotropy [8]. Some methods for adaptive morphology, such as the line-shaped or rectangular structuring elements presented by Verdú-Monedero et al. [21] or the continuous PDE-based morphology presented by Breuß et al. [6], use the LST components implicitly or explicitly, but without using the anisotropy information it contains. Only the Elliptical Adaptive Structuring Elements (EASE) method $[11,12]$ takes advantage of this property of the LST.

The methods listed above are all presented for the so called flat case, where structuring elements are defined by sets of points rather than functions. While flat morphology may certainly be highly useful it does have limitations, and it is natural to consider an extension to the non-flat case, where structuring elements become structuring functions. Non-flat adaptive mathematical morphology is far from common in literature, but some work have been published. Bouaynaya and Schonfeld $[4,5]$ have presented a base for adaptive structuring functions. The non-flat case has also been considered in non-local morphology by Salembier [16] and Velasco-Forero and Angulo [20]. Angulo and Velasco-Forero [3] have used structuring functions based on random walks, and Curić and Luengo Hendriks [10] have presented a method for salience-adaptive morphology based on paraboloidal structuring functions. Moreover, Quadratic Structuring Functions (QSFs) have been used by Angulo [1] as a base for flat adaptive morphology by thresholding them at a given level.

This work builds on the previously presented EASE method [11,12], presenting a strategy for non-flat mathematical morphology where QSFs are set from the Local Structure Tensor.

\subsection{Contribution}

It has been shown that Quadratic Structuring Functions (QSFs) play a similar role to morphology as Gaussian functions do to standard convolution filtering, i.e. QSFs are to the morphological $(\max ,+)$ and $(\min ,+)$ algebras what the Gaussian function is to the standard $(+, \times)$ algebra used in linear filtering $[7$, 19]. QSFs are thereby or particular interest - and importance - to mathematical morphology.

As QSFs are paraboloids with elliptical level contours, the previously presented concept of Elliptical Adaptive Structuring Elements (EASE) [12] can be 
quite naturally extended into the non-flat case, following the general theoretical work presented by Bouaynaya and Schonfeld $[4,5]$. This article presents a method that sets QSFs based on the information contained in the LST, building on previous work [12] but enabling non-flat structuring functions. The presented strategy yields a novel straight-forward approach to non-flat structure-based adaptive morphology based on QSFs, which can be quite efficiently implemented. Moreover; only two user-set parameters are required, which makes the method easy to use.

\section{Method}

\subsection{Adaptive Structuring Functions}

Let $f$ denote an image with values $f(\mathbf{x}) \in[0,1]$ defined for points $\mathbf{x} \in \mathcal{D}(f)$ and, following the theoretical work by Bouaynaya et al. $[4,5]$, let

$$
s_{\mathbf{x}}(\mathbf{u})=s[\mathbf{x}](\mathbf{u}-\mathbf{x}) \quad \forall \mathbf{u} \in \mathcal{D}\left(s_{\mathbf{x}}\right) \subseteq \mathcal{D}(f)
$$

denote an adaptive structuring function for a point $\mathbf{x}$. Note that both $\mathbf{x}$ and $\mathbf{u}$ are given in global coordinates. The notation $s[\mathbf{x}]$ denotes that the function $s$ itself, rather than just its values, varies with $\mathbf{x}$. The corresponding so called reflected (or transposed) structuring function, used for defining proper morphological operations, is defined as

$$
s_{\mathbf{x}}^{*}(\mathbf{u})=s_{\mathbf{u}}(\mathbf{x})=s[\mathbf{u}](\mathbf{x}-\mathbf{u}) .
$$

The complete set of structuring functions $\left\{s_{\mathbf{x}} \mid \mathbf{x} \in \mathcal{D}(f)\right\}$ is known as a Structuring Element Map (SEM) for the image. This SEM also implicitly contains the reflected (or transposed) structuring functions.

The morphological erosion and dilation for the adaptive case are then given by (see Ref. [4]):

$$
\begin{aligned}
\varepsilon_{s}(f) & =\bigwedge_{\mathbf{u} \in \mathcal{D}\left(s_{\mathbf{x}}\right)}\left\{f(\mathbf{u})-s_{\mathbf{x}}(\mathbf{u})\right\}, \\
\delta_{s}(f) & =\bigvee_{\mathbf{u} \in \mathcal{D}\left(s_{\mathbf{x}}^{*}\right)}\left\{f(\mathbf{u})+s_{\mathbf{x}}^{*}(\mathbf{u})\right\} .
\end{aligned}
$$

Given that the SEM remains constant over the operations, these definitions ensure that the erosion and dilation are adjunct - a property often overseen within adaptive morphology, as noted by Roerdink [15]. The opening and closing can now be properly defined as

$$
\begin{aligned}
& \gamma_{s}(f)=\left(\delta_{s} \circ \varepsilon_{s}\right)(f)=\delta_{s}\left(\varepsilon_{s}(f)\right), \\
& \varphi_{s}(f)=\left(\varepsilon_{s} \circ \delta_{s}\right)(f)=\varepsilon_{s}\left(\delta_{s}(f)\right) .
\end{aligned}
$$

In practice the dilation $\delta_{s}(f)$ can be calculated by the following algorithm, which goes through all points $\mathbf{x}$ and updates the value at point $\mathbf{u}$ in $\delta_{s}(f)$ when $f(\mathbf{x})+$ $s_{\mathbf{x}}(\mathbf{u})$ yields a higher value (similar to previous usage for the flat case, see e.g. Ref. [13]):

$$
\left(\delta_{s}(f)\right)(\mathbf{u}) \longleftarrow \bigvee\left\{\left(\delta_{s}(f)\right)(\mathbf{u}), f(\mathbf{x})+s_{\mathbf{x}}(\mathbf{u})\right\}, \forall \mathbf{u} \in s_{\mathbf{x}}, \forall \mathbf{x} \in \mathcal{D}(f) .
$$

We now simply need to define our SEM. 


\subsection{The Local Structure Tensor}

As demonstrated in previous work [12], the Local Structure Tensor (LST) can be used to define elliptical adaptive structuring elements. The LST $\mathbf{T}(\mathbf{x})$ can be constructed from the image gradient by

$$
\mathbf{T}(\mathbf{x})=\left(\begin{array}{ll}
T_{11} & T_{12} \\
T_{12} & T_{22}
\end{array}\right)(\mathbf{x})=G_{\sigma} *\left(\nabla f(\mathbf{x}) \nabla^{\mathrm{T}} f(\mathbf{x})\right),
$$

where $\nabla=\left(\frac{\partial}{\partial x_{1}} \frac{\partial}{\partial x_{2}}\right)^{\mathrm{T}}$ and $G_{\sigma}$ is a Gaussian kernel with standard deviation $\sigma$ [8]. The image gradient can be estimated by applying standard gradient filters on a slightly smoothed version of the input image. The parameter $\sigma$ sets the scale for which the LST should be representative, and can be set implicitly by defining a radial bandwidth $r_{w}$ for the filter (so that $G_{\sigma}$ decreases to half of its maximum value at distance $r_{w}$ from its center), i.e.

$$
\sigma=\frac{r_{w}}{\sqrt{2 \ln 2}} .
$$

The eigenvalues $\lambda_{1}(\mathbf{x})$ and $\lambda_{2}(\mathbf{x})\left(\lambda_{1}(\mathbf{x}) \geq \lambda_{2}(\mathbf{x})\right)$ and corresponding eigenvectors $\mathbf{e}_{1}(\mathbf{x})$ and $\mathbf{e}_{2}(\mathbf{x})$ of $\mathbf{T}(\mathbf{x})$ hold information about structures (edges) in the image. Eigenvalues can be interpreted based on Table 1, while $\mathbf{e}_{2}(\mathbf{x})$ represents the direction of the smallest variation [8].

Table 1: Interpretation of the eigenvalues $\lambda_{1}$ and $\lambda_{2}$ of the LST $\mathbf{T}$

\begin{tabular}{ll}
\hline$\lambda_{1} \approx \lambda_{2} \gg 0$ & $\begin{array}{l}\text { No dominant direction } \\
\text { (edge crossing or point) }\end{array}$ \\
$\lambda_{1} \gg \lambda_{2} \approx 0$ & Strong dominant direction (edge) \\
$\lambda_{1} \approx \lambda_{2} \approx 0$ & No dominant direction (no edge) \\
\hline
\end{tabular}

\subsection{Flat Structuring Elements}

Previous work $[12,11]$ has defined flat elliptical adaptive structuring elements. The axes $a(\mathbf{x})$ and $b(\mathbf{x})$ are set from the eigenvalues of $\mathbf{T}(\mathbf{x})$ by the expressions

$$
a(\mathbf{x})=\frac{\lambda_{1}(\mathbf{x})}{\lambda_{1}(\mathbf{x})+\lambda_{2}(\mathbf{x})} \cdot M, \quad b(\mathbf{x})=\frac{\lambda_{2}(\mathbf{x})}{\lambda_{1}(\mathbf{x})+\lambda_{2}(\mathbf{x})} \cdot M,
$$

where $M$ denotes the maximum allowed semi-major axis. Numerical stability is addressed by adding a small positive number (i.e. machine epsilon) to the eigenvalues. The orientation $\theta(\mathbf{x})$ is retrieved from the corresponding eigenvectors 
by

$$
\theta(\mathbf{x})=\left\{\begin{array}{cc}
\arctan \left(\frac{e_{2, x_{2}}(\mathbf{x})}{e_{2, x_{1}}(\mathbf{x})}\right), & e_{2, x_{1}}(\mathbf{x}) \neq 0, \\
\pi / 2, & e_{2, x_{1}}(\mathbf{x})=0
\end{array}\right.
$$

where $e_{2, x_{1}}(\mathbf{x})$ and $e_{2, x_{2}}(\mathbf{x})$ denote the components of the eigenvector $\mathbf{e}_{2}(\mathbf{x})$. The resulting structuring elements range dynamically from lines of length $M$ where $\lambda_{1}(\mathbf{x}) \gg \lambda_{2}(\mathbf{x}) \approx 0$, i.e. near strong dominant edges in the data, to disks with radius $\frac{M}{2}$ where $\lambda_{1}(\mathbf{x}) \approx \lambda_{2}(\mathbf{x})$, i.e. where no single direction represents the local image structure.

\subsection{Quadratic Structuring Functions}

The change from flat structuring elements to Quadratic Structuring Functions (QSFs) is quite straight-forward, yet yields a substantial change: the LST is still used to calculate $a, b$, and $\theta$, but the parameters are now used to set QSFs rather than flat elliptical structuring elements. The result is a method for nonflat adaptive morphology based on a well known method for estimating structure in images.

Given $a, b$, and $\theta$ we define the quadratic structuring function (with elliptical level contours)

$$
\begin{aligned}
s_{\mathbf{x}}(\mathbf{u})=-\frac{1}{2}\left(\left(\frac{\left(x_{1}-u_{1}\right) \cos \theta+\left(x_{2}-u_{2}\right) \sin \theta}{a}\right)^{2}\right. \\
\left.+\left(\frac{\left(x_{1}-u_{1}\right) \sin \theta-\left(x_{2}-u_{2}\right) \cos \theta}{b}\right)^{2}\right) \\
=-\frac{1}{2}(\mathbf{u}-\mathbf{x})^{T} \mathbf{R}\left(\begin{array}{cc}
a^{-2} & 0 \\
0 & b^{-2}
\end{array}\right) \mathbf{R}^{T}(\mathbf{u}-\mathbf{x}) \\
=-\frac{1}{2 M^{2}}(\mathbf{u}-\mathbf{x})^{T} \mathbf{R}\left(\begin{array}{cc}
\alpha^{-2} & 0 \\
0 & \beta^{-2}
\end{array}\right) \mathbf{R}^{T}(\mathbf{u}-\mathbf{x})
\end{aligned}
$$

where

$$
\mathbf{R}=\left(\begin{array}{rr}
\cos \theta & -\sin \theta \\
\sin \theta & \cos \theta
\end{array}\right), \quad \alpha=\frac{\lambda_{1}}{\lambda_{1}+\lambda_{2}}, \quad \beta=\frac{\lambda_{2}}{\lambda_{1}+\lambda_{2}} .
$$

For numerical stability, Eqs. (12) or (13) should be used for setting the QSFs. To avoid division by zero in the case when $b=0, a$ and $b$ (which are measured in pixel units) can be increased by $\frac{1}{2}$. If image function values within the range $[0,1]$ can be assumed, a non-flat structuring function $s_{\mathbf{x}}$ will not cause any change where $s_{\mathbf{x}}<-1$. The values of the QSFs can therefore be precomputed on a fixed spatial support $\mathcal{D}\left(s_{\mathbf{x}}\right)$ large enough to ensure $s_{\mathbf{x}}(\mathbf{u}) \leq-1 \forall \mathbf{u} \notin \mathcal{D}\left(s_{\mathbf{x}}\right)$.

Equation (14) clearly shows how $M$ becomes a scale factor for the structuring functions. It should be noted that the set $\left\{\mathbf{u} \mid s_{\mathbf{x}}(\mathbf{u}) \geq-\frac{1}{2}\right\}$ yields the flat elliptical structuring element in the previous section. 


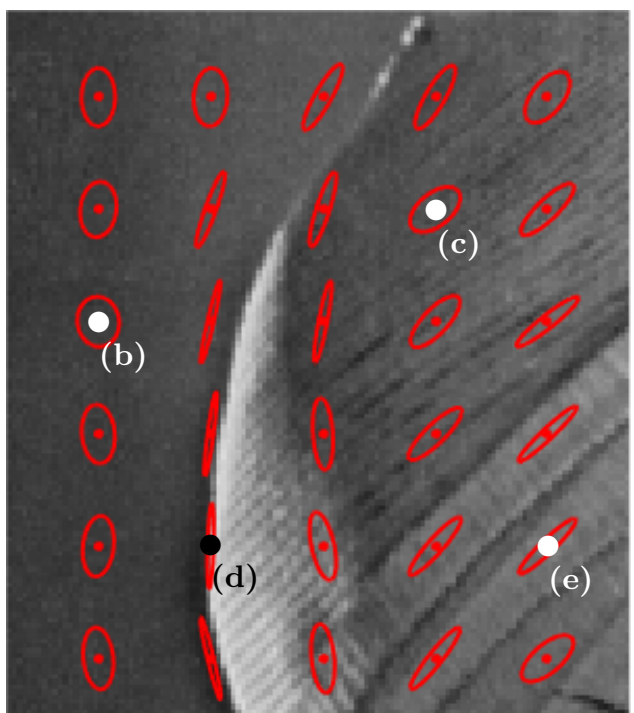

(a)

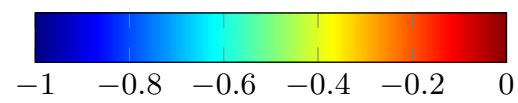

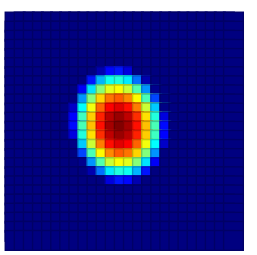

(b)

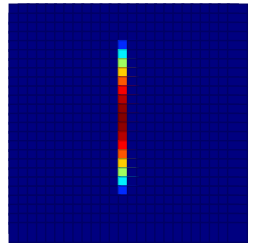

(d)

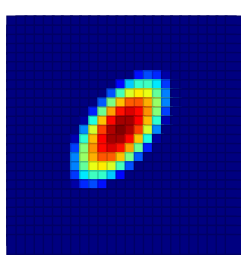

(c)

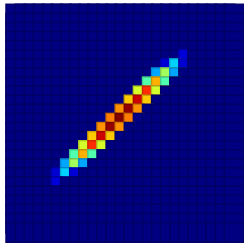

(e)

Fig. 1: Detailed examples of structuring functions. (a): An original image with the contours $s_{\mathbf{x}}=-\frac{1}{2}$ drawn in red for a subset of the SEM. (b)-(e): The QSFs for the corresponding points marked in (a).

\section{$3 \quad$ Results}

\subsection{Structuring Function Shapes}

Figure 1 shows a close-up of an input image and a subset of its SEM for $M=$ $r_{w}=8$ (Fig. 1a). The red ellipses in Fig. 1a show the contours of $s_{\mathbf{x}}=-\frac{1}{2}$ for the selected subset of points, and are thereby equivalent to the flat ellipses in Sect. 2.3. Four detailed examples of the resulting QSFs, corresponding to the points marked with letters are presented in detail in Figs. 1b-1e.

The close connection to the flat elliptical structuring elements is obvious, and it is clear that the structuring functions follow the image structure well. One potential issue, which does not show up in this particular subset of structuring functions but may be easily anticipated, may appear as a result from going from a continuous definition to a discrete domain of pixels: in the case of a QSF with a domain in the shape of a line which is not aligned with the axes, the maximum of the expression may end up in-between pixels. Consider, for instance, a small shift of the orientation of the structuring function in Fig. 1d. The values in the outer pixels would soon end up in between the pixel positions, as the structuring function is very thin. This would make the implemented structuring function shorter than the theoretical. 


\subsection{Comparison to Flat EASE}

Figure 2 compares the presented QSF method to flat EASE. Looking at the top and left parts of the result for flat EASE (Fig. 2b), sharp edges are introduced by the flat structuring elements while the quadratic structuring functions leave a much more smooth result (Figs. 2c-2f). It should be noted that as the impact of a QSF decreases with distance to its middle point, the presented method obviously need a larger value of $M$ than the flat elliptical structuring elements in order to achieve a similar level of change in the image.

The effect of different scales of the morphological operations are clear: as the scale for QSFs is increased (Figs. 2c-2f), so are the changes to the original image. The result for $M=2$ is more or less identical to the original image (Fig. 2c), but as $M$ is increased the result becomes more and more similar to (yet still more smooth than) the more severe changes resulting from the flat operation (compare Fig. 2f with Fig. 2b).

\subsection{Morphological Operations}

Figure 3 shows examples of morphological operations based on the presented QSFs. The erosion and opening successfully enhances dark lines and edges, while the dilation and closing do the same for bright directional features. This is most evident in the hat, hair, and feathers. Also note how the mouth changes in different ways in the erosion and dilation.

More examples of adaptive openings and closings by the presented method are presented in Fig. 4. The dark structures around the windows in the image on the first row (original in the left column) are clearly enhanced by the opening (middle row), while the closing (right column) instead links the bright spots along the left edge of the roof into a continuous bright line. In the second row the opening removes bright features such as the whiskers, while darks regions such as the mouth are enhanced. The closing instead makes the whiskers show more clearly.

The third row demonstrates how the opening enhances the dark branches. Note how the structuring functions change from anisotropic to isotropic as branches cross, which means that there is no obvious direction to follow. If desired (which may depend on the specific application), this effect could be countered by changing the size of the structuring functions based on the magnitudes of the eigenvalues (which will both be high at crossings).

In the fourth row, the linking properties of the presented method are clear: the opening enhances the dark rail while the closing connects the originally slightly separated parts of the bright train. Finally, the last row demonstrates the use of the method in a specific practical case: the opening enhances a crack in steel, making it easier to find and classify the crack. Note that the parameter $r_{w}$ sets the scale for the structures to align to, which means that both $M$ and $r_{w}$ need to be related to the size of the gaps to bridge. 


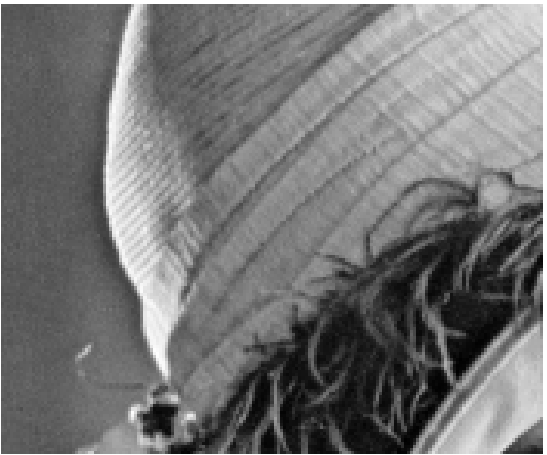

(a)

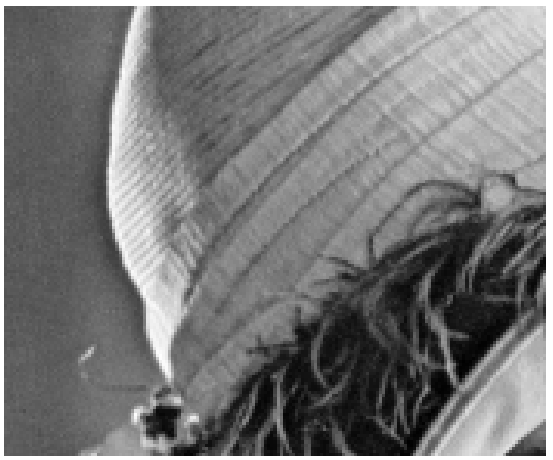

(c)

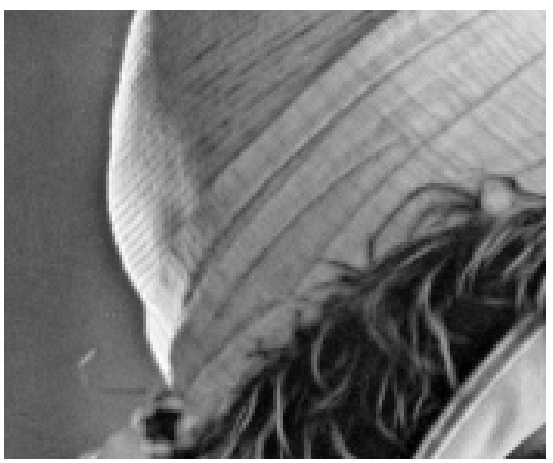

(e)

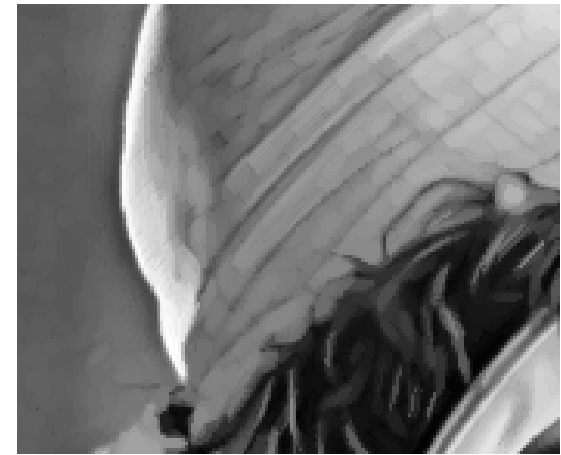

(b)

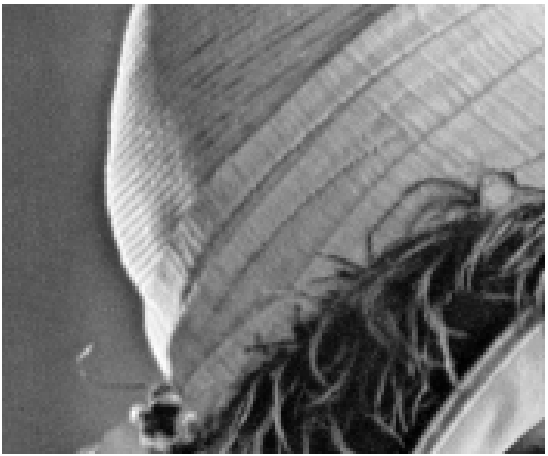

(d)

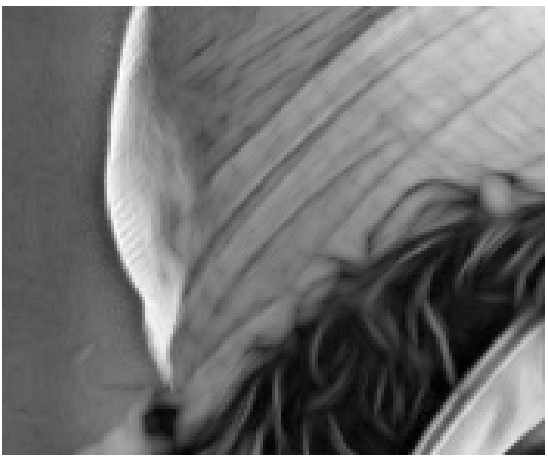

(f)

Fig. 2: Top row: close-up (size $175 \times 145$ pixels) of an original image (a) and its opening by flat EASE with $M=r_{w}=4$ (b). Second and third rows: Openings by the presented QSFs for $M=\{2,4,8,16\}, r_{w}=4$ (c,d,e, and f). 


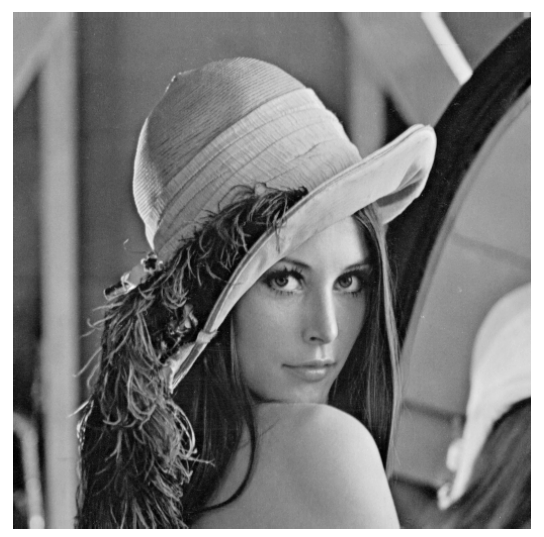

(a)

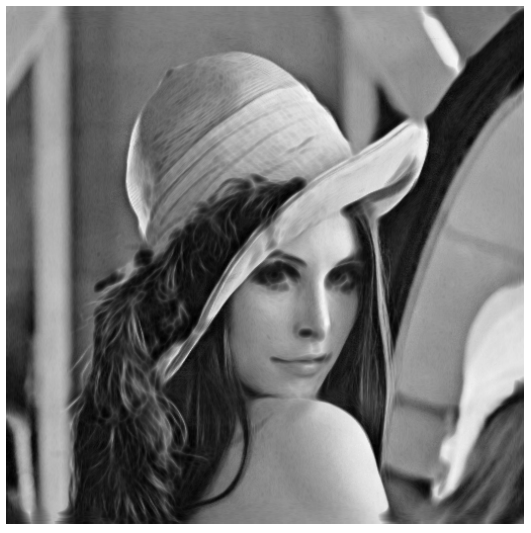

(b)

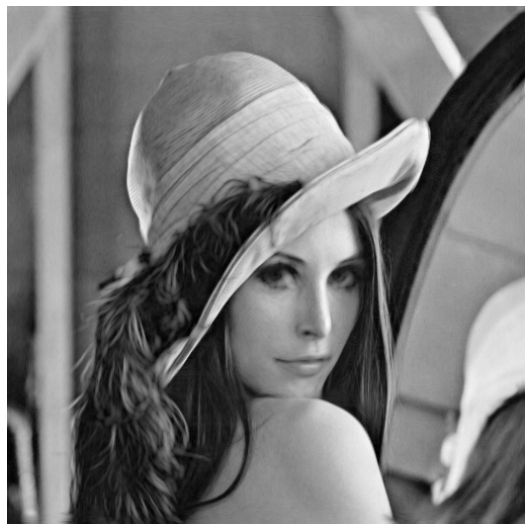

(d)

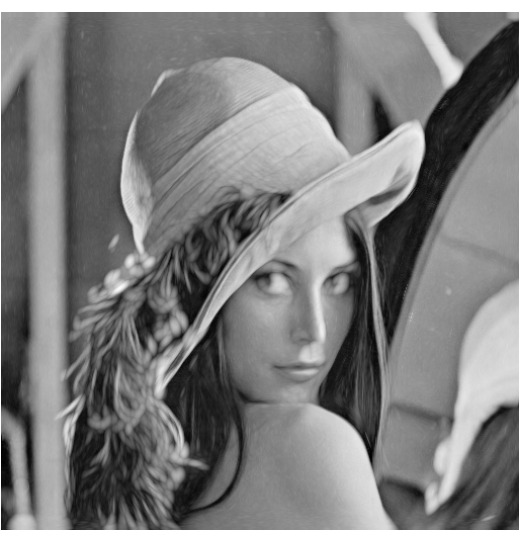

(c)

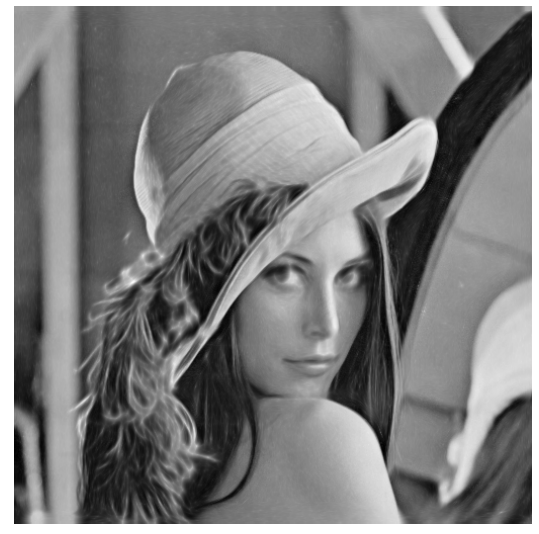

(e)

Fig. 3: An original image (size $512 \times 512$ pixels) and examples of the erosion (b), dilation (c), opening (d), and closing (e) with $M=16, r_{w}=8$. 

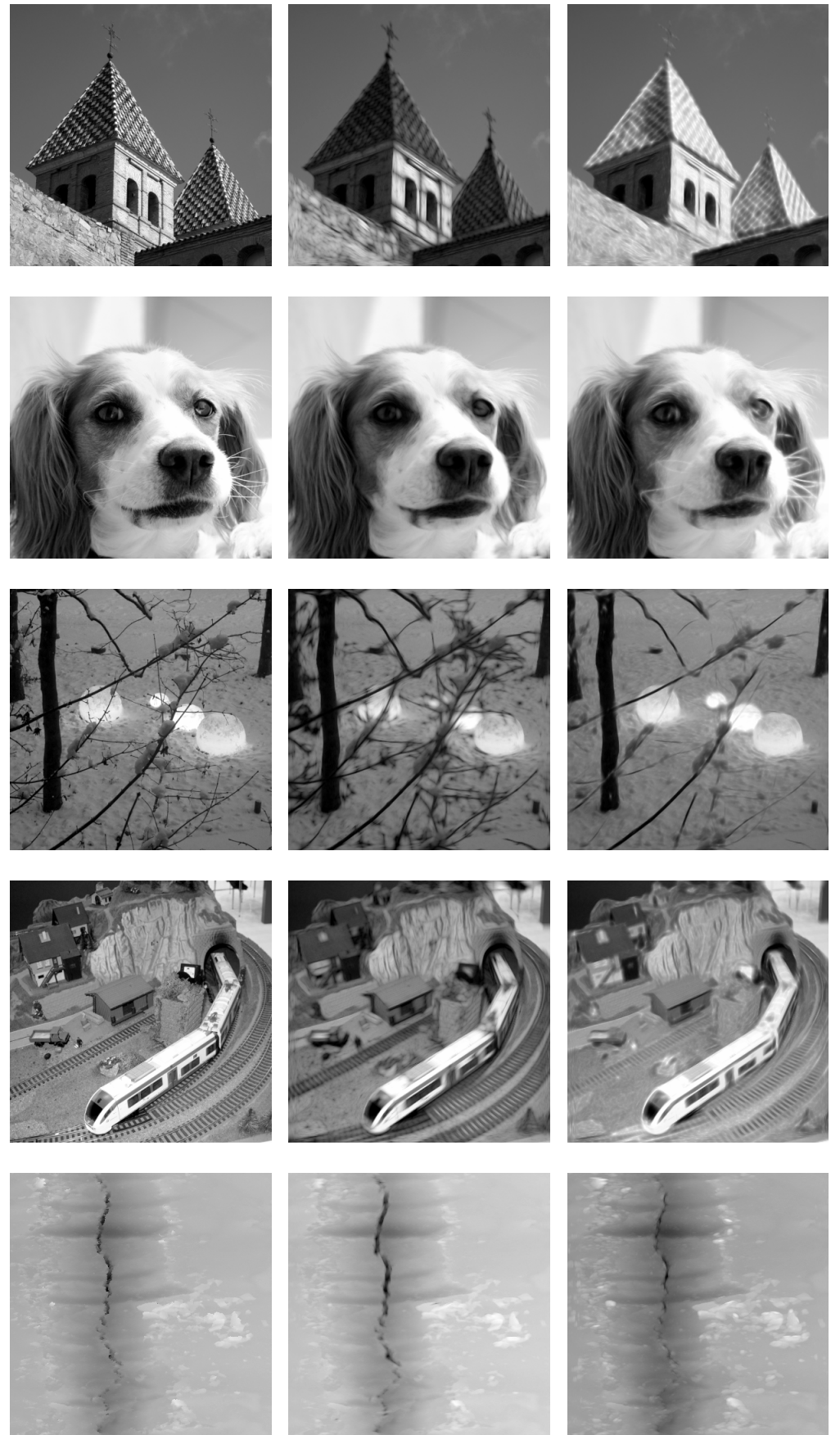

Fig. 4: Five original images of size $400 \times 400$ pixels (left column), and their openings (middle column) and closings (right column) using $M=16, r_{w}=8$. 


\section{Conclusion}

In this work an approach for interpreting the LST into well defined QSFs in a straight-forward manner has been investigated. The results show that the presented method successfully enhances edges and lines, and adapts well to structure. It builds on the previously presented EASE method, with the important contribution of allowing non-flat adaptive morphology.

The strategy does not only demonstrate a mathematically solid method for non-flat morphological filtering, based on well known theory, but presents a method which can be quite easily implemented with a low number of user-set parameters (the scale for the LST and the scale for the QSF, and possibly the level of prefiltering) which are easily related to measures in the image. The implementation can be done quite efficiently by pre-calculating the weights of the quadratic structuring functions. There are some issues to consider when going from continuous theory to discrete practice, however, as discussed in Sect. 3.1.

The presented method demonstrates a solid interpretation of the LST which yields well defined structuring functions, but there are of course other ways in which the LST could be converted into QSFs. Future work should investigate this direction further. In particular, the relation to the covariance matrix (which under some assumptions is equivalent to the LST) and the Mahalanobis distance should be pursued. A quantitative comparison to other methods based on adaptive structuring functions should also be conducted.

\section{Acknowledgment}

The author would like to thank Dr. Jesús Angulo for highly appreciated input, which inspired this work.

\section{References}

1. J. Angulo. Morphological bilateral filtering and spatially-variant adaptive structuring functions. In Mathematical Morphology and Its Applications to Image and Signal Processing, pages 212-223. Springer, 2011.

2. J. Angulo and S. Velasco-Forero. Structurally adaptive mathematical morphology based on nonlinear scale-space decompositions. Image Analysis 8 Stereology, 30(2):111-122, 2011.

3. J. Angulo and S. Velasco-Forero. Stochastic morphological filtering and bellmanmaslov chains. In Mathematical Morphology and Its Applications to Signal and Image Processing, pages 171-182. Springer, 2013.

4. N. Bouaynaya and D. Schonfeld. Spatially variant morphological image processing: theory and applications. In Proceedings of SPIE, volume 6077, pages 673-684, 2006.

5. N. Bouaynaya and D. Schonfeld. Theoretical foundations of spatially-variant mathematical morphology part II: Gray-level images. IEEE Transactions on Pattern Analysis and Machine Intelligence, 30(5):837-850, 2008.

6. M. Breuß, B. Burgeth, and J. Weickert. Anisotropic continuous-scale morphology. Pattern Recognition and Image Analysis, pages 515-522, 2007. 
7. B. Burgeth and J. Weickert. An explanation for the logarithmic connection between linear and morphological system theory. International Journal of Computer Vision, 64(2-3):157-169, 2005.

8. L. Cammoun, C.A. Castaño-Moraga, E. Muñoz-Moreno, D. Sosa-Cabrera, B. Acar, M.A. Rodriguez-Florido, A. Brun, H. Knutsson, and J.P. Thiran. A review of tensors and tensor signal processing. In Tensors in Image Processing and Computer Vision, pages 1-32. Springer, 2009.

9. V. Ćurić, A. Landström, M.J. Thurley, and C.L. Luengo Hendriks. Adaptive mathematical morphology - a survey of the field. Pattern Recognition Letters, 47(0):18 - 28, 2014. Advances in Mathematical Morphology.

10. V. Curić and C.L. Luengo Hendriks. Salience-based parabolic structuring functions. In Mathematical Morphology and Its Applications to Signal and Image Processing, pages 183-194. Springer, 2013.

11. A. Landström. Elliptical Adaptive Structuring Elements for Mathematical Morphology. Doctoral thesis, Luleå University of Technology, Sweden, 2014.

12. A. Landström and M.J. Thurley. Adaptive morphology using tensor-based elliptical structuring elements. Pattern Recognition Letters, 34(12):1416-1422, 2013.

13. R. Lerallut, É. Decencière, and F. Meyer. Image filtering using morphological amoebas. In Mathematical Morphology: 40 Years On, pages 13-22, 2005.

14. P. Maragos and C. Vachier. Overview of adaptive morphology: trends and perspectives. In 16th IEEE International Conference on Image Processing (ICIP), pages 2241-2244. IEEE, 2009.

15. J.B.T.M. Roerdink. Adaptivity and group invariance in mathematical morphology. In 16th IEEE International Conference on Image Processing (ICIP), pages 22532256. IEEE, 2009.

16. P. Salembier. Study on nonlocal morphological operators. In Image Processing (ICIP), 2009 16th IEEE International Conference on, pages 2269-2272. IEEE, 2009.

17. F.Y. Shih and S. Cheng. Adaptive mathematical morphology for edge linking. Information sciences, 167(1):9-21, 2004.

18. O. Tankyevych, H. Talbot, P. Dokládal, and N. Passat. Direction-adaptive greylevel morphology. application to $3 \mathrm{~d}$ vascular brain imaging. In 16th IEEE International Conference on Image Processing (ICIP), pages 2261-2264. IEEE, 2009.

19. R. Van Den Boomgaard, L. Dorst, S. Makram-Ebeid, and John Schavemaker. Quadratic structuring functions in mathematical morphology. In Mathematical morphology and its applications to image and signal processing, pages 147-154. Springer, 1996.

20. S. Velasco-Forero and J. Angulo. On nonlocal mathematical morphology. In Mathematical Morphology and Its Applications to Signal and Image Processing, pages 219-230. Springer, 2013.

21. R. Verdú-Monedero, J. Angulo, and J. Serra. Anisotropic morphological filters with spatially-variant structuring elements based on image-dependent gradient fields. IEEE Transactions on Image Processing, 20(1):200-212, 2011. 